\title{
Tratamento cirúrgico da rigidez pós-traumática do cotovelo por via posterior ampla*
}

\section{Surgical Treatment of Post-traumatic Elbow Stiffness by Wide Posterior Approach}

\author{
Mauro E.C. Gracitelli ${ }^{10}$ César L.B. Guglielmetti ${ }^{1}$ Caio A.S. Botelho ${ }^{1}$ Eduardo A. Malavolta ${ }^{1}$ \\ Jorge H. Assunção ${ }^{1}$ Arnaldo A. Ferreira Neto ${ }^{1}$ \\ 1 Grupo de Ombro e Cotovelo, Instituto de Ortopedia e \\ Traumatologia, Hospital das Clínicas, Faculdade de Medicina, \\ Universidade de São Paulo, São Paulo, SP, Brasil \\ Endereço para correspondência Mauro E. C. Gracitelli, PhD, Rua \\ Dr. Ovídio Pires de Campos, 333, $3^{\circ}$ andar, Cerqueira Cesar, São Paulo, \\ SP, 05403-010, Brasil (e-mail: mgracitelli@gmail.com).
}

Rev Bras Ortop 2020;55(5):570-578.

\section{Resumo \\ Palavras-chave \\ - articulação do cotovelo \\ - contratura \\ - rigidez pós- traumática \\ - liberação da cápsula articular \\ - resultado do tratamento}

Objetivo Demonstrar os resultados clínicos e a taxa de complicações da liberação cirúrgica por via única posterior no tratamento da rigidez pós-traumática de cotovelo. Métodos Estudo prospectivo, com pacientes submetidos a cirurgia entre maio de 2013 e junho de 2018 em um único centro. Foi realizado acesso ao cotovelo por via posterior. O seguimento dos pacientes foi feito por uma equipe de terapia ocupacional, e eles foram submetidos a um protocolo de reabilitação padronizado, com órteses estáticas progressivas e dinâmicas. O desfecho primário foi a amplitude de flexoextensão do cotovelo após 6 meses.

Resultados Um total de 26 pacientes completaram o seguimento mínimo de 6 meses. A média de flexoextensão do cotovelo, ao final de 6 meses, foi de $98,3^{\circ} \pm 22,0^{\circ}$, com um ganho de amplitude de $40,0^{\circ} \pm 14,0^{\circ}$ em relação ao pré-operatório $(p<0,001)$. A média de ganho relativo de flexoextensão, ao final de 6 meses, foi de $51,7 \% \pm 17,1 \%(p<0,001)$. A média de pronossupinação, ao final de 6 meses, foi de $129,0^{\circ} \pm 42,7^{\circ}(p<0,001)$. Metade dos casos apresentava rigidez moderada e grave no pré-operatório, contra 7,7\% aos 6 meses de pós-operatório $(p<0,001)$. A pontuação nos instrumentos Mayo Elbow Performance Score (MEPS) e Disabilities of the Arm, Shoulder and Hand (DASH) apresentou melhora estatisticamente significativa em relação ao pré-operatório, atingindo 74,4 $\pm 16,8$ pontos e $31,7 \pm 21,9$ pontos, respectivamente. A escala visual analógica (EVA) não apresentou diferença estatisticamente significativa em relação ao pré-operatório $(p=0,096)$. Complicações foram observadas em $6(23 \%)$ pacientes, não sendo necessária nova abordagem cirúrgica em nenhum paciente.

Conclusões A liberação cirúrgica do cotovelo associada a protocolo de reabilitação é técnica segura, com resultados satisfatórios e baixa taxa de complicações.

\footnotetext{
Trabalho desenvolvido no Grupo de Ombro e Cotovelo, Instituto de Ortopedia e Traumatologia, Hospital das Clínicas, Faculdade de Medicina, Universidade de São Paulo (HCFMUSP), São Paulo, SP, Brasil.
}

recebido

31 de Março de 2019 aceito 23 de Julho de 2019
DOI https://doi.org/

10.1055/s-0039-1700827. ISSN 0102-3616.
Copyright $\odot 2020$ by Sociedade Brasileira License terms de Ortopedia e Traumatologia. Published by Thieme Revinter Publicações Ltda, Rio de Janeiro, Brazil 
Abstract

\section{Keywords}

- elbow joint

- contracture

- post-traumatic stiffness

- joint capsule release

- treatment outcome
Objective To demonstrate the clinical outcomes and complication rates of the surgical release with a single posterior approach in the treatment of post-traumatic elbow stiffness.

Methods A prospective study with patients submitted to surgery between May 2013 and June 2018 in a single center. The access to the elbow was made through the posterior approach. The patients were followed up by an occupational therapy team, and were submitted to a standardized rehabilitation protocol, with static progressive orthoses and dynamic orthoses. The primary outcome was the range of flexionextension of the elbow after 6 months.

Results A total of 26 patients completed the minimum follow-up of 6-months. The mean range of flexion-extension of the elbow at the end of 6 months was of $98.3 \pm 22.0^{\circ}$, with an amplitude gain of $40.0 \pm 14.0^{\circ}$ in relation to the pre-operative period $(p<0.001)$. The average flexion-extension gain at the end of 6 months was of $51.7 \% \pm 17.1 \%(p<0.001)$. The mean pronosupination at the end of 6 months was of $129.0 \pm 42.7^{\circ}(p<0.001)$. Half of the cases had moderate and severe stiffness in the pre-operative period, compared with $7.7 \%$ at 6 months post-operatively $(p<0.001)$. The mean score for the Mayo Elbow Performance Score (MEPS) and Disabilities of the Arm, Shoulder and Hand (DASH) instruments was of $74.4 \pm 16.8$ points and $31.7 \pm 21.9$ points respectively $(p<0.001$ for both). The visual analogue scale (VAS) score presented no statistically significant difference compared to the preoperative period $(p=0.096)$. Complications were observed in $6(23 \%)$ patients, and no new surgical procedures were necessary.

Conclusions The surgical release of the elbow associated with a rehabilitation protocol is a safe technique, with satisfactory results and low rate of complications.

\section{Introdução}

O cotovelo tem a função de movimentar e posicionar a mão no espaço. ${ }^{1}$ Sua amplitude de movimento (ADM) é essencial para a maioria das atividades diárias. É aceito que a ADM funcional do cotovelo é entre $-30^{\circ}$ de extensão e $130^{\circ}$ de flexão, e de $100^{\circ}$ de pronossupinação, sendo $50^{\circ}$ para cada direção. ${ }^{2}$ Atividades contemporâneas, como utilizar o computador e o telefone celular, aparentemente exigem respectivamente uma maior pronação e flexão do que as definidas na ADM funcional. ${ }^{3}$

A rigidez pós-traumática do cotovelo (RPTC) é definida como a perda de qualquer grau de movimento após um trauma; porém, a maioria dos autores considera como indicação de tratamento apenas quando ocorre perda da ADM funcional. ${ }^{4-6}$ A verdadeira incidência da RPTC não é conhecida. Aproximadamente $12 \%$ das lesões no cotovelo resultam em contraturas que requerem algum tipo de liberação cirúrgica. ${ }^{7,8}$

O tratamento da RPTC apresenta inúmeros desafios. Devido à variabilidade das lesões, das causas e da sintomatologia, existem grandes dificuldades na homogeneização dos protocolos e na comparação de resultados entre os diferentes tratamentos. Pacientes com diferentes níveis de gravidade, de tempo de rigidez, de dor e de artrose, evoluem de maneiras distintas com tratamentos semelhantes. ${ }^{9-12}$
Para os pacientes com RPTC sem consolidação viciosa, pseudartrose, material de síntese intra-articular ou ossificação heterotópica $(\mathrm{OH})$, e sem melhora com a fisioterapia convencional, existem duas opções de tratamento: ${ }^{13,14}$ liberação cirúrgica ou protocolos de reabilitação com terapia ocupacional, com mobilização leve e progressiva associada ao uso de órteses.

Diversas técnicas cirúrgicas já foram descritas para o tratamento da RPTC, desde as mais agressivas, com liberação extensa associada ao fixador externo, ${ }^{15}$ até as minimamente invasivas ${ }^{5}$ e por via artroscópica. ${ }^{16}$ Os autores relatam bons resultados, com ganho significativo da função e ADM do cotovelo, com taxas de complicação que variam de $0 \%$ a $88 \%{ }^{6}$ Porém, existem poucos estudos com bom nível de evidência, com apenas duas séries prospectivas de casos. ${ }^{14,17}$

Nosso objetivo é demonstrar os resultados clínicos e a taxa de complicação de uma técnica de liberação aberta, por via posterior única, sem o uso de fixador externo, seguida de um protocolo de reabilitação padronizado. 0 desfecho primário deste estudo é avaliar a ADM de flexoextensão dos pacientes. Os desfechos secundários são avaliar o resultado clínico, de acordo com a escala Mayo Elbow Performance Score (MEPS), ${ }^{18}$ o questionário Disabilities of the Arm, Shoulder and Hand (DASH) 19 e a escala visual analógica (EVA) de dor, o ganho absoluto e relativo da ADM de flexoextensão, ${ }^{20}$ a flexão e extensão máximas, a ADM de pronossupinação, e a taxa de complicações. 


\section{Materiais e Métodos}

\section{Desenho do Estudo}

Realizamos um estudo prospectivo envolvendo 33 pacientes com RPTC. Os pacientes foram operados entre maio de $2013 \mathrm{e}$ junho de 2018 em um único centro. As cirurgias foram realizadas por dois cirurgiões, que seguiram a mesma técnica cirúrgica. O protocolo foi aprovado pela Comissão de Ética de nosso serviço, e registrado na Plataforma Brasil.

\section{Participantes}

Os critérios de inclusão foram idade entre 18 e 65 anos, histórico prévio de trauma que evoluiu com rigidez articular do cotovelo, ADM de flexoextensão do cotovelo menor do que $100^{\circ}$ ou extensão máxima menor do que $-30^{\circ}$, ou flexão máxima menor do que $130^{\circ}$, com mais de 6 meses do trauma inicial. Não foram incluídos os pacientes com bloqueio articular (arco de movimento igual a $0^{\circ}$ ), lesão neurológica do membro superior acometido, doença mental ou incapacidade para compreender os questionários, infecção prévia no cotovelo, e $\mathrm{OH}$.

\section{Intervenção}

As cirurgias tiveram início após realização de bloqueio do plexo braquial interescalênico associado à anestesia geral sem colocação de garrote. Os pacientes foram posicionados em decúbito dorsal. Todos os pacientes receberam antibioticoprofilaxia com cefazolina $1 \mathrm{~g}$ por via intravenosa de $8 \mathrm{em}$
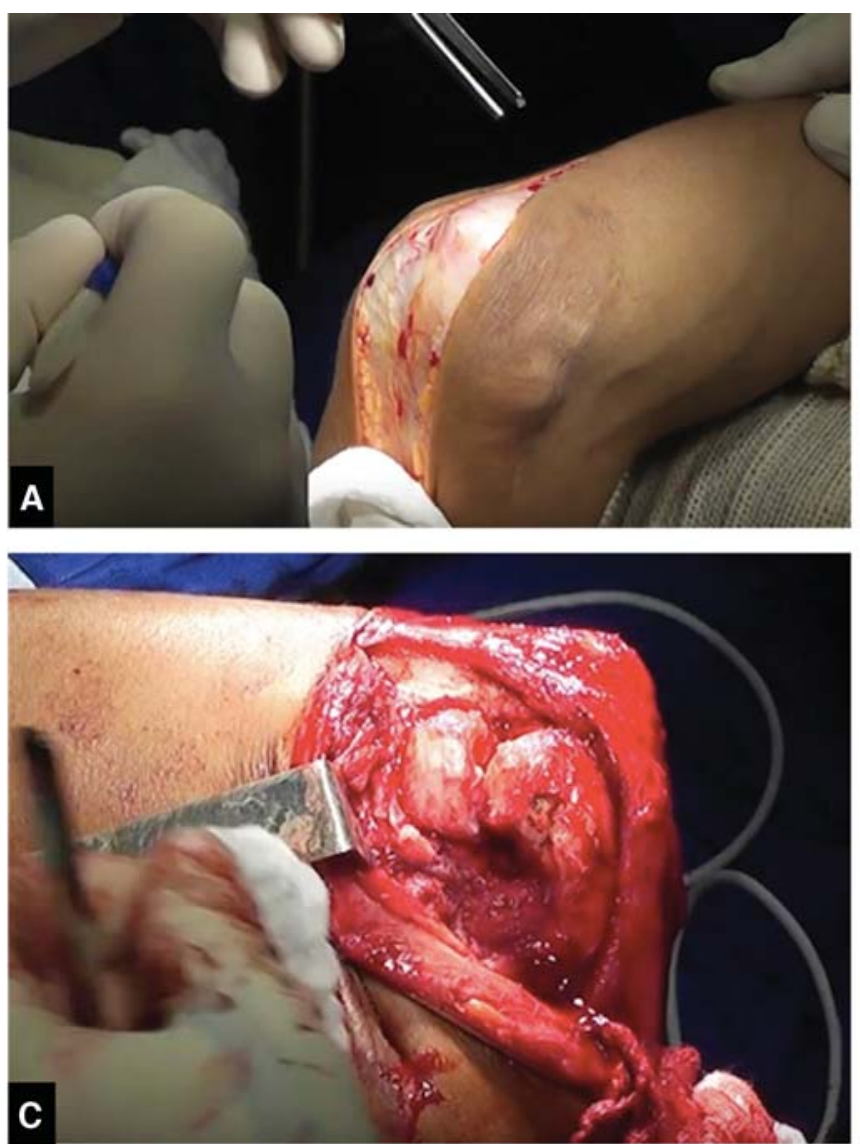

8 horas, por um período de 24 horas, sendo a primeira dose administrada 30 minutos antes do início da cirurgia.

Foi realizado acesso ao cotovelo por via posterior ampla de $12 \mathrm{~cm}$ a $15 \mathrm{~cm}$, desviando lateralmente da ponta do olécrano. O nervo ulnar foi identificado, liberado e protegido, seguido de dissecção lateral e medial do músculo tríceps braquial até o úmero, sem desinserção do tendão tricipital. A massa extensora do antebraço foi elevada da cápsula anterior, seguida de liberação de sua inserção umeral em conjunto com o ligamento colateral lateral. A porção posterior do ligamento colateral medial foi liberada. Realizamos a liberação de aderências articulares entre o músculo tríceps braquial e o úmero, excisão de osteófitos periarticulares, desbridamento e limpeza da fossa olecraniana, e liberação da cápsula anterior da porção distal do úmero. Após liberação completa e ganho total da amplitude de movimento, foi realizada a reinserção dos ligamentos colateral lateral e medial com pontos transósseos com fios não absorvíveis Ethibond Excel 2-0 (Johnson\&Johnson, New Brunswick, NJ, EUA). Antes do fechamento por planos, foi checada a manutenção da amplitude de movimento e colocado dreno a vácuo em todos os casos, em volta da articulação, de modo a não ficar interposto nela. Foi realizada transposição subcutânea anterior do nervo ulnar. O curativo foi realizado com gaze e enfaixamento seguido de imobilização em extensão máxima com tala gessada anterior. A técnica utilizada é demonstrada no vídeo suplementar ao artigo e na - Figura 1.
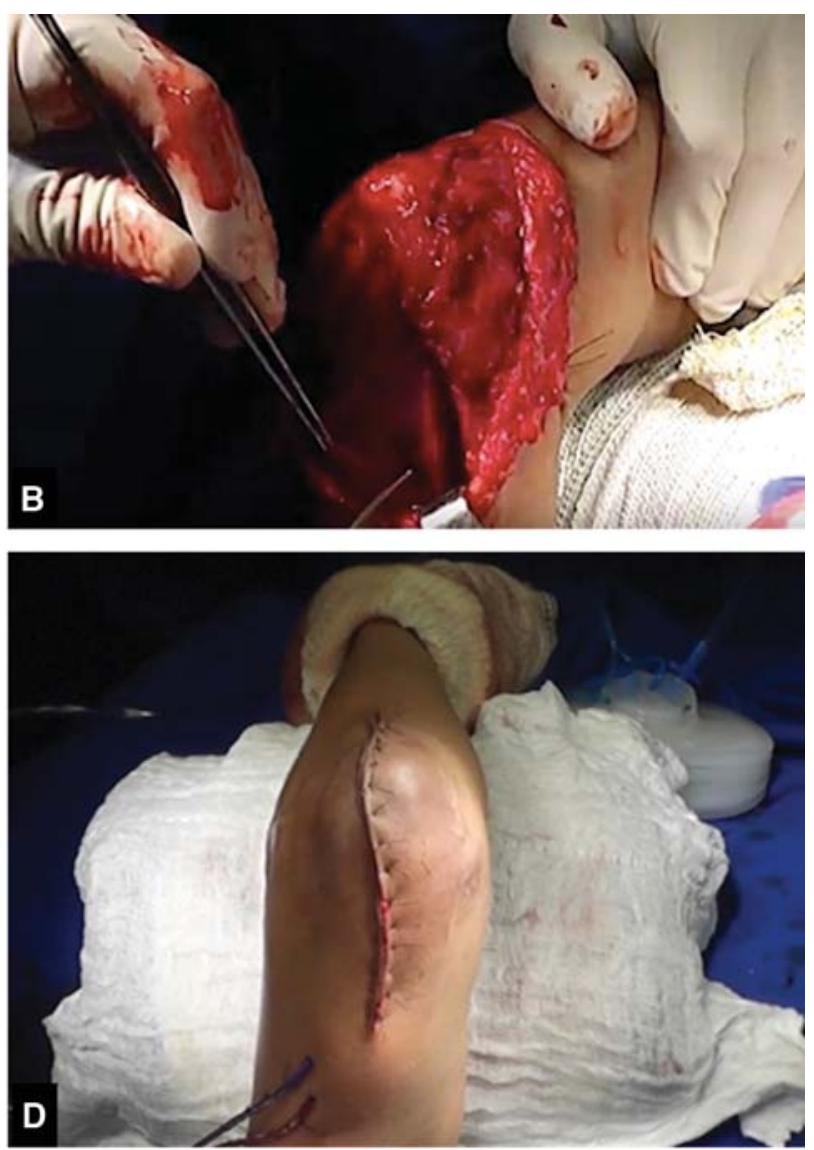

Fig. 1 Imagem da técnica cirúrgica. (A) via posterior ampla; (B) dissecção medial e localização do nervo ulnar; (C) dissecção lateral e visualização da cabeça do rádio e capítulo; (D) fechamento e aspecto final com colocação de dreno a vácuo. 
Os pacientes permaneceram três dias internados para controle da dor, edema (manutenção do membro elevado) e mobilização precoce. $\mathrm{O}$ seguimento deles foi feito pela equipe de terapia ocupacional, e eles foram submetidos a um protocolo de reabilitação padronizado. No primeiro dia pós-operatório, foi realizada uma órtese de polietileno em extensão máxima com a equipe de terapia ocupacional, que foi utilizada em período integral, sendo retirada apenas para a higienização e para os exercícios. Os pacientes foram submetidos a movimento passivo contínuo (MPC) diariamente por uma hora durante o período de internação, e duas vezes na semana após a alta hospitalar, durante as sessões de terapia ocupacional. Os pacientes fizeram exercícios ativos livres e assistidos durante o dia, realizando extensão, flexão, pronação e supinação, e foram estimulados a realizar atividades diárias sem carga com o membro. Durante as sessões de terapia ocupacional, os pacientes aprenderam a fazer os exercícios em casa, visando não ultrapassar quatro pontos de dor de acordo com a EVA. Eles foram instruídos a praticar diariamente os exercícios em casa, a cada 2 horas por 5 a 10 minutos. 0 dreno a vácuo foi retirado após 48 horas da cirurgia, sendo realizado o MPC com o uso do dreno. Na primeira semana, os pacientes utilizaram a órtese estática em extensão, retirando-a para a higienização e para os exercícios feitos em casa. Após este período, retiraramna apenas para dormir. Após 1 mês da cirurgia, eles começaram a utilizar a órtese dinâmica para ganho de flexão, 3 vezes ao dia, por períodos de 30 minutos. As órteses foram reajustadas conforme a necessidade durante as sessões de terapia ocupacional. As órteses são demonstradas na - Figura 2.

\section{Desfechos}

O desfecho primário do estudo foi a ADM de flexoextensão do cotovelo após seis meses da cirurgia.

Os desfechos secundários foram as escalas MEPS e EVA, o questionário DASH, a ADM de pronossupinação, o ganho relativo da $A D M$, as incidências de complicações clínicas e radiográficas, e a necessidade de nova abordagem cirúrgica. Os resultados da escala MEPS foram categorizados em excelentes ( $>90$ pontos), bons (entre 75 e 89 pontos), regulares (entre 60 e 74 ) e ruins ( $<60$ pontos).

\section{Variáveis Analisadas}

A avaliação da ADM de flexoextensão do cotovelo foi realizada em graus com o auxílio de um goniômetro manual por um avaliador treinado, não participante da equipe de reabilitação, com o paciente em posição ortostática e com ombro elevado a $90^{\circ}$. Para a pronossupinação, o paciente foi avaliado com o ombro em adução e rotação neutra, e com o cotovelo fletido a $90^{\circ}$. Com a mão, o paciente segurou um bastão.

Outras variáveis clínicas foram avaliadas, tais como: características gerais (gênero, idade, dominância e lado acometido); hábitos (tabagismo); doenças pré-existentes (diabetes, hipertensão arterial e hipotireoidismo); presença de fraturas prévias; e tratamento prévio realizado (cirúrgico ou conservador).

Antes do início do tratamento, foram realizadas tomografia computadorizada e radiografias de frente e perfil do cotovelo em todos os pacientes. Foram avaliados a presença de corpos livres e de osteófitos, o grau de degeneração articular, ${ }^{21}$ e a presença de material de síntese.

As complicações foram anotadas de acordo com a sua ocorrência, e o número total delas e de pacientes foram registrados separadamente. A reabordagem cirúrgica e o tipo de cirurgia realizada também foram registrados.

\section{Análise Estatística}

Avaliamos a normalidade dos dados por meio do teste de Shapiro-Wilk, e a homogeneidade, por meio do teste de Levene. Apresentamos as variáveis contínuas em médias e desvio padrão. As variáveis categóricas foram apresentadas em valores absoluto e percentual.

Para a análise da evolução ao longo do tempo dos desfechos clínicos, foi realizado o teste de Friedman. A análise dos dados categóricos foi realizada com o teste exato de Fischer ou do qui-quadrado.

Valores de $p<0,05$ foram considerados estatisticamente significativos.

Para a análise dos dados, utilizou-se o programa Statistical Package for the Social Sciences (SPSS, IBM Corp., Armonk, NY, EUA), versão 21.0.
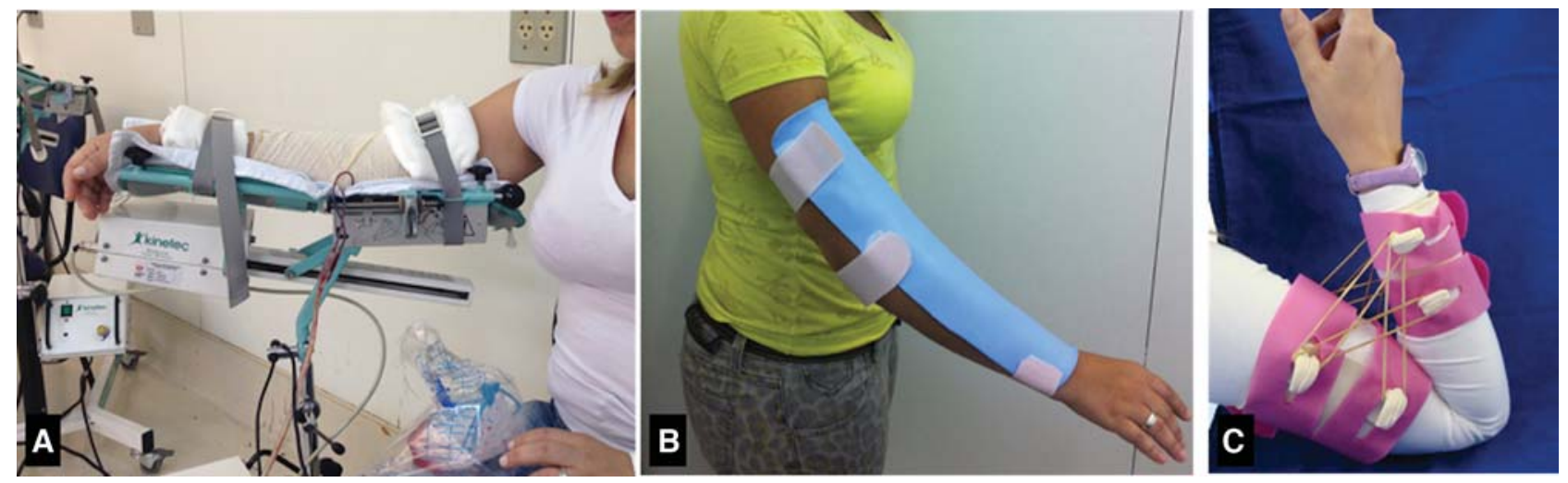

Fig. 2 Caso ilustrando o (A) aparelho de movimento passivo contínuo (MPC); (B) a órtese estática para ganho da extensão; e (C) a órtese dinâmica para ganho da flexão. 
574 Tratamento cirúrgico da rigidez pós-traumática do cotovelo Gracitelli et al.

Tabela 1 Características clínicas dos pacientes submetidos ao tratamento cirúrgico para liberação do cotovelo

\begin{tabular}{|c|c|}
\hline Características clínicas & Casos \\
\hline \multicolumn{2}{|l|}{ Gênero, $n$ (\%) } \\
\hline Masculino & $17(65,4)$ \\
\hline Feminino & $9(34,6)$ \\
\hline \multicolumn{2}{|l|}{ Idade, anos } \\
\hline Média (desvio padrão) & $37,3(11,3)$ \\
\hline \multicolumn{2}{|l|}{ Tabagismo, $n(\%)$} \\
\hline Sim & $2(7,7)$ \\
\hline Ex-tabagista & $3(11,5)$ \\
\hline Não & $21(80,8)$ \\
\hline \multicolumn{2}{|l|}{ Diabetes, $n(\%)$} \\
\hline Sim & $2(7,7)$ \\
\hline Não & $24(92,3)$ \\
\hline \multicolumn{2}{|c|}{ Problemas no trabalho, $n$ (\%) } \\
\hline Sim & $7(26,9)$ \\
\hline Não & $19(73,1)$ \\
\hline \multicolumn{2}{|l|}{ Lado acometido, $n(\%)$} \\
\hline Direito & $14(53,8)$ \\
\hline Esquerdo & $12(46,2)$ \\
\hline \multicolumn{2}{|c|}{ Lado dominante acometido, $n$ (\%) } \\
\hline Sim & $14(53,8)$ \\
\hline Não & $12(46,2)$ \\
\hline \multicolumn{2}{|l|}{ Fratura prévia, $n$ (\%) } \\
\hline Sim & $19(73,1)$ \\
\hline Não & $7(26,9)$ \\
\hline \multicolumn{2}{|l|}{ Cirurgia prévia, $n$ (\%) } \\
\hline Sim & $8(30,8)$ \\
\hline Não & $18(69,2)$ \\
\hline
\end{tabular}

Nota: *Os dados contínuos são apresentados como média e desvio padrão, e os dados categóricos, como números absolutos, com as porcentagens entre parênteses.

\section{Resultados}

Foram operados 33 pacientes com RPTC. Destes, seis foram excluídos por perda de seguimento. Foram analisados 26 pacientes, com 6 meses de seguimento. Os dados gerais da amostra podem ser vistos na - Tabela 1. Os dados radiográficos estão expostos na - Tabela 2.

As causas que levaram à RPTC foram: sete casos por fratura do úmero distal; cinco pacientes por fratura isolada da cabeça do rádio; quatro casos por tríade terrível; quatro pacientes por fratura do coronoide; dois casos por luxação do cotovelo isolada; e quatro pacientes por outras causas (um por ferimento corto-contuso, dois por imobilização prolongada por contusão, e um por contusão do cotovelo associada a traumatismo cranioencefálico).
Tabela 2 Características radiográficas dos pacientes submetidos ao tratamento cirúrgico para liberação do cotovelo

\begin{tabular}{|l|l|}
\hline Características radiográficas & Casos \\
\hline Presença de material de síntese, $n(\%)$ & \\
\hline Sim & $8(30,8)$ \\
\hline Não & $18(69,2)$ \\
\hline Cabeça do rádio, $n$ (\%) & \\
\hline Sem deformidade & $16(61,5)$ \\
\hline Com deformidade & $7(26,9)$ \\
\hline Ausente & $2(7,7)$ \\
\hline Prótese & $1(3,8)$ \\
\hline Corpos livres, $n(\%)$ & \\
\hline Sim & $10(38,5)$ \\
\hline Não & $16(61,5)$ \\
\hline Osteófitos, $n$ (\%) & \\
\hline Sim & $13(50,0)$ \\
\hline Não & $13(50,0)$ \\
\hline
\end{tabular}

Nota: * Os dados categóricos são apresentados como números absolutos, com as porcentagens entre parênteses.

A média da ADM de flexoextensão do cotovelo, ao final de 6 meses de seguimento, foi de $98,3^{\circ} \pm 22,0^{\circ}$, com um ganho de ADM de flexoextensão do cotovelo de $40,0^{\circ} \pm 14,0^{\circ}$ em relação ao pré-operatório, com diferença estatisticamente significativa $(p<0,001)$. A média de ganho relativo da ADM de flexoextensão do cotovelo, ao final de 6 meses de seguimento, foi de $51,7 \% \pm 17,1 \%$, com diferença estatisticamente significaiva na análise temporal $(p<0,001)$.

A média da ADM de pronossupinação do cotovelo, no préoperatório, foi de $115,2^{\circ} \pm 52,3^{\circ}$ e, ao final de 6 meses de seguimento, de $129,0^{\circ} \pm 42,7^{\circ}$, com diferença estatisticamente significativa na análise temporal $(p<0,001)$.

Metade dos casos apresentava rigidez moderada e grave no pré-operatório, contra apenas 7,7\% aos 6 meses de pósoperatório $(p<0,001)$. Os resultados categóricos são apresentados na - Tabela 3. Os valores das médias de ADM do cotovelo e do ganho de ADM são apresentados na - Tabela $4 \mathrm{e}$ nas -Figuras 3 e 4.

Todas as médias finais da ADM(flexão, extensão, pronação e supinação) apresentaram melhora estatisticamente significativa na análise temporal, e são apresentadas na - Tabela 5.

A média da pontuação pelas escalas MEPS e DASH, ao final dos 6 meses de seguimento, foi de $74,4 \pm 16,8$ pontos e de $31,7 \pm 21,9$ pontos, respectivamente. Ambas apresentaram diferença estatisticamente significativa na análise temporal $(p<0,001)$. A EVA não apresentou diferença estatisticamente significativa na análise temporal $(p=0,096)$. Os valores das médias são apresentados na -Tabela $\mathbf{6}$ e na -Figura $\mathbf{5}$.

Obtivemos 15 pacientes com resultado excelente ou bom, e 11 com resultado regular ou ruim, de acordo com a escala MEPS, com diferença estatisticamente significativa em relação ao pré-operatório $(p=0,011)$. Os dados são apresentados na -Tabela 3. 
Tratamento cirúrgico da rigidez pós-traumática do cotovelo Gracitelli et al. 575

Tabela 3 Características radiográficas dos pacientes submetidos ao tratamento cirúrgico para liberação do cotovelo

\begin{tabular}{|c|c|c|c|}
\hline & $\begin{array}{l}\text { Pré- } \\
\text { operatório }\end{array}$ & 6 meses & $\begin{array}{l}\text { Valor } \\
\text { de } p\end{array}$ \\
\hline \multicolumn{4}{|c|}{ Grau da rigidez } \\
\hline 1 & $1(3,8)$ & $18(69,2)$ & \\
\hline II & $12(46,2)$ & $6(23,1)$ & \\
\hline III & $9(34,6)$ & $2(7,7)$ & \\
\hline IV & $4(15,4)$ & $0(0)$ & $<0,0001$ \\
\hline \multicolumn{4}{|c|}{ Grau da artrose } \\
\hline 0 & $5(19,2)$ & $4(16,0)$ & \\
\hline 1 & $8(30,8)$ & $8(32,0)$ & \\
\hline 2 & $11(42,3)$ & $9(36,0)$ & \\
\hline 3 & $2(7,7)$ & $4(16,0)$ & 0,811 \\
\hline \multicolumn{4}{|c|}{$\begin{array}{l}\text { Mayo Elbow } \\
\text { Performance Score } \\
\text { (MEPS) categórica }\end{array}$} \\
\hline Excelente & $0(0)$ & $4(15,4)$ & \\
\hline Bom & $5(19,2)$ & $11(42,3)$ & \\
\hline Regular & $8(30,8)$ & $7(26,9)$ & \\
\hline Ruim & $13(50,0)$ & $4(15,4)$ & 0,011 \\
\hline
\end{tabular}

Nota: *Os dados categóricos são apresentados como números absolutos, com as porcentagens entre parênteses.

Tabela 4 Resultados da amplitude de movimento (ADM) de flexoextensão, do ganho absoluto e relativo de ADM de flexoextensão, e da ADM de pronossupinação ao longo do seguimento

\begin{tabular}{|c|c|c|c|}
\hline & \multicolumn{2}{|l|}{ Casos } & \multirow[t]{2}{*}{ Valor de $p$} \\
\hline & Média & $\begin{array}{l}\text { Desvio } \\
\text { padrão }\end{array}$ & \\
\hline \multicolumn{4}{|c|}{ ADM de flexoextensão $\left({ }^{\circ}\right)$} \\
\hline Inicial & 58,4 & 22,7 & \\
\hline 6 semanas & 78,0 & 22,5 & \\
\hline 3 meses & 89,4 & 26,0 & \\
\hline 6 meses & 98,3 & 22,0 & $<0,001$ \\
\hline \multicolumn{4}{|c|}{ Ganho de ADM ( $\left.{ }^{\circ}\right)$} \\
\hline 6 semanas & 15,6 & 23,2 & \\
\hline 3 meses & 31,9 & 20,9 & \\
\hline 6 meses & 40,0 & 14,0 & $<0,001$ \\
\hline \multicolumn{4}{|c|}{ Ganho relativo de ADM (\%) } \\
\hline 6 semanas & 16,4 & 28,9 & \\
\hline 3 meses & 39,5 & 24,0 & \\
\hline 6 meses & 51,7 & 17,1 & $<0,001$ \\
\hline \multicolumn{4}{|c|}{ ADM pronossupinação $\left({ }^{\circ}\right)$} \\
\hline Inicial & 115,2 & 52,3 & \\
\hline 6 semanas & 117,4 & 42,7 & \\
\hline 3 meses & 124,3 & 43,8 & \\
\hline 6 meses & 129,0 & 42,7 & $<0,001$ \\
\hline
\end{tabular}

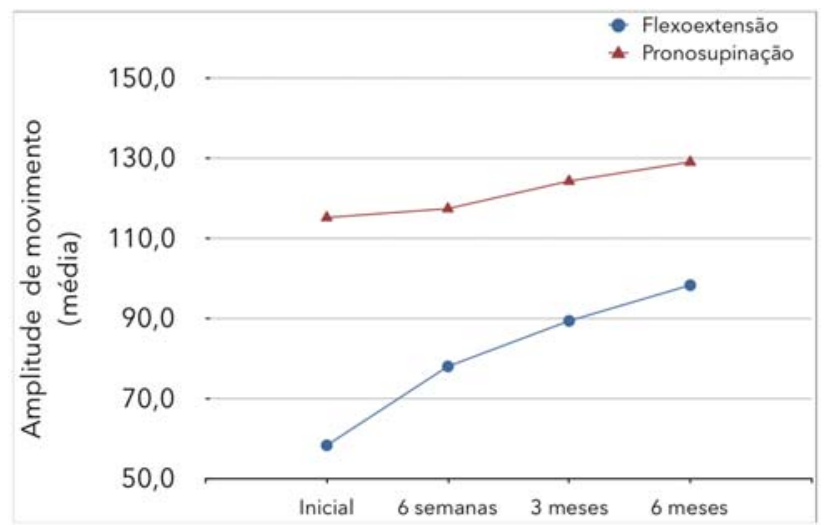

Fig. 3 Resultados da amplitude de movimento de flexoextensão e pronossupinação ao longo do seguimento.

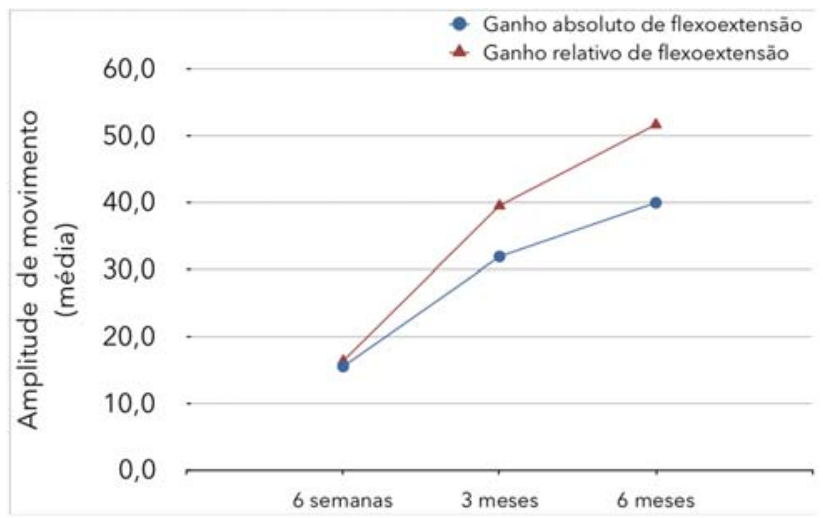

Fig. 4 Resultados do ganho da amplitude de movimento absoluta e relativa de flexoextensão ao longo do seguimento.

A ressecção da cabeça do rádio foi realizada em dois casos devido a deformidades, e em um paciente foi retirada uma artroplastia da cabeça do rádio. Dois pacientes já apresentavam ressecção prévia da cabeça do rádio, totalizando cinco pacientes com ausência da cabeça do rádio no pós-operatório. Esses pacientes apresentavam uma média de 66 pontos pela escala MEPS ( $\pm 18,8$ pontos), média inferior à dos pacientes sem deformidade da cabeça do rádio $(81 \pm 11,9$ pontos), mas sem diferença estatisticamente significativa. Não observamos diferença na análise univariada de subgrupo para as demais variáveis clínicas e características da lesão. A presença de osteófitos (no coronoide, na tróclea ou no olécrano) também não demonstrou diferença estatisticamente significativa no pós-operatório, apesar de o grupo sem osteófitos ter obtido melhores resultados na escala MEPS e na ADM de flexoextensão (78,1 $\pm 17,0$ versus $70,8 \pm 16,3$, e $100,1 \pm 20,2$ versus $95,7 \pm 24,3$, respectivamente).

As seguintes complicações foram observadas em 6 (23\%) pacientes: neuropraxia transitória do nervo ulnar (4 casos); subluxação da cabeça do rádio (1 caso); infecção superficial (1 caso); e $\mathrm{OH}$ (1 caso). A OH ocorreu no tendão distal do tríceps, e o paciente apresentou flexão final de $90^{\circ}$ e extensão de $-15^{\circ}$, não desejando novo procedimento cirúrgico. Os quatro casos de neuropraxia transitória do nervo ulnar apresentaram melhora completa em até cinco meses de pós-operatório, não necessitando de nova abordagem. 
Tabela 5 Resultados das médias de flexão, extensão, pronação e supinação ao longo do seguimento

\begin{tabular}{|c|c|c|c|}
\hline & \multicolumn{2}{|l|}{ Casos } & \multirow[t]{2}{*}{ Valor de $p$} \\
\hline & Média & Desvio padrão & \\
\hline \multicolumn{4}{|l|}{ Flexão $\left(^{\circ}\right)$} \\
\hline Inicial & 99,3 & 24,8 & \\
\hline 6 semanas & 101,7 & 20,3 & \\
\hline 3 meses & 108,8 & 21,0 & \\
\hline 6 meses & 116,0 & 17,6 & $<0,001$ \\
\hline \multicolumn{4}{|l|}{ Extensão $\left({ }^{\circ}\right)$} \\
\hline Inicial & $-40,9$ & 20,9 & \\
\hline 6 semanas & $-30,2$ & 17,7 & \\
\hline 3 meses & $-19,4$ & 11,1 & \\
\hline 6 meses & $-17,7$ & 10,6 & $<0,001$ \\
\hline \multicolumn{4}{|l|}{ Pronação $\left({ }^{\circ}\right)$} \\
\hline Inicial & 58,1 & 27,6 & \\
\hline 6 semanas & 55,4 & 24,7 & \\
\hline 3 meses & 59,0 & 24,0 & \\
\hline 6 meses & 62,5 & 24,1 & 0,017 \\
\hline \multicolumn{4}{|l|}{ Supinação $\left({ }^{\circ}\right)$} \\
\hline Inicial & 57,5 & 29,4 & \\
\hline 6 semanas & 62,4 & 26,3 & \\
\hline 3 meses & 66,1 & 26,6 & \\
\hline 6 meses & 67,5 & 26,0 & $<0,001$ \\
\hline
\end{tabular}

Tabela 6 Resultados das escalas funcionais dos pacientes submetidos à liberação cirúrgica ao longo do seguimento

\begin{tabular}{|c|c|c|c|}
\hline & \multicolumn{2}{|l|}{ Casos } & \multirow[t]{2}{*}{ Valor de $p$} \\
\hline & Média & $\begin{array}{l}\text { Desvio } \\
\text { padrão }\end{array}$ & \\
\hline \multicolumn{4}{|c|}{$\begin{array}{l}\text { Mayo Elbow Performance } \\
\text { Score (MEPS) }\end{array}$} \\
\hline Inicial & 54,4 & 20,1 & \\
\hline 3 meses & 70,2 & 20,1 & \\
\hline 6 meses & 74,4 & 16,8 & $<0,001$ \\
\hline \multicolumn{4}{|c|}{$\begin{array}{l}\text { Disabilities of the Arm, } \\
\text { Shoulder and Hand (DASH) }\end{array}$} \\
\hline Inicial & 47,5 & 19,2 & \\
\hline 3 meses & 37,1 & 23,4 & \\
\hline 6 meses & 31,7 & 21,9 & $<0,001$ \\
\hline \multicolumn{4}{|c|}{$\begin{array}{l}\text { Escala Visual } \\
\text { Analógica (EVA) de dor }\end{array}$} \\
\hline Inicial & 5,1 & 3,1 & \\
\hline 6 semanas & 4,4 & 3,0 & \\
\hline 3 meses & 4,0 & 2,9 & \\
\hline 6 meses & 4,1 & 2,5 & 0,096 \\
\hline
\end{tabular}

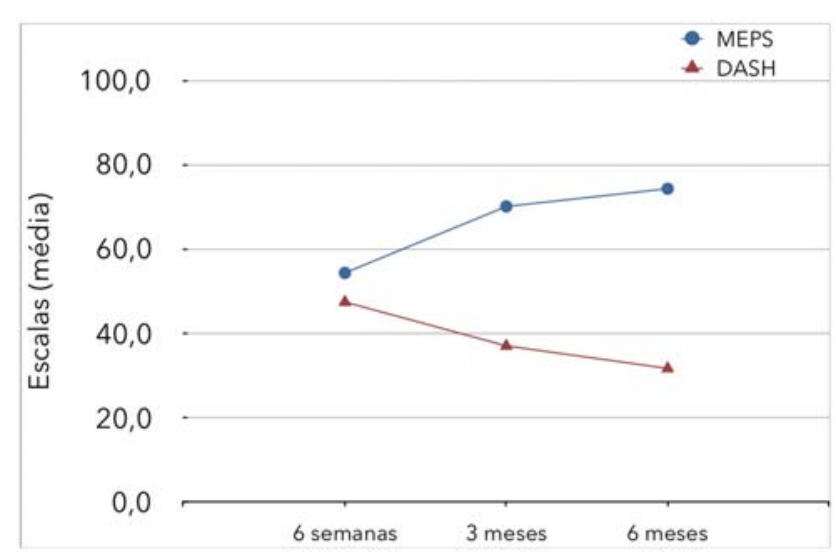

Fig. 5 Resultados das escalas funcionais Mayo Elbow Performance Score (MEPS) e Disabilities of the Arm, Shoulder and Hand (DASH) ao longo do seguimento.

O paciente com infecção superficial apresentou melhora com antibioticoterapia oral.

Nenhum paciente evoluiu com lesão grave de partes moles ou infecção profunda. Nenhum paciente necessitou de novo procedimento cirúrgico em razão das complicações.

\section{Discussão}

Este estudo teve como objetivo avaliar a liberação cirúrgica do cotovelo seguida de um protocolo de reabilitação padronizado para os pacientes com RPTC que já haviam sido submetidos ao tratamento fisioterápico convencional sem sucesso. $O$ tratamento da rigidez do cotovelo é tecnicamente complexo, com riscos moderados de complicações. A indicação do tratamento cirúrgico ainda é controversa, principalmente devido à ausência de estudos comparativos para a RPTC. Em nossos critérios de indicação, levamos em consideração o desejo e a motivação do paciente em melhorar sua amplitude de movimento, assim como um limite mínimo de $\mathrm{ADM}$ de flexoextensão do cotovelo menor do que $100^{\circ}$ ou extensão máxima menor do que $-30^{\circ}$, ou flexão máxima menor do que $130^{\circ}$, com mais de 6 meses do trauma inicial.

A técnica estudada apresentou resultados bons e excelentes em mais da metade dos pacientes operados, com uma média de $\mathrm{ADM}$ de flexoextensão de $98,3^{\circ}$, semelhante à média encontrada na revisão sistemática de Kodde et al. ${ }^{6}$ de $103^{\circ}$ para as liberações abertas.

Em relação ao ganho da ADM de flexoextensão do cotovelo após 6 meses de tratamento, demonstramos um ganho de $40^{\circ}$, semelhante aos valores apresentados por Higgs et al. ${ }^{14}$ Koh et al. ${ }^{22}$ e Miyazaki et al. ${ }^{23}$ Já Ayadi et al. ${ }^{24}$ observaram ganho de $51^{\circ}$ da ADM de flexoextensão em série de pacientes submetidos à liberação cirúrgica aberta, mas apenas $18 \%$ dos pacientes alcançaram a ADM funcional, enquanto em nosso estudo observamos que $42 \%$ dos pacientes atingiram a ADM funcional aos 6 meses de seguimento. Devido a esse achado, é importante ressaltar que, na comparação dos resultados de ganho da ADM entre estudos, o ganho da ADM é inversamente proporcional à $\mathrm{ADM}$ pré-operatória. ${ }^{22}$ Assim, em estudos em que a ADM inicial é menor, os pacientes terão 
uma tendência a um maior ganho de ADM pós-tratamento. Tal fato torna a comparação entre os resultados dos estudos de difícil execução e interpretação, podendo levar a conclusões equivocadas. Na tentativa de contornar esse problema, Cauchoix e Deburge ${ }^{20}$ criam uma fórmula levando em consideração o ganho possível pré-tratamento e o ganho atingido após o tratamento, sendo o resultado em porcentagem; eles chamaram isso de ganho relativo da ADM. Nosso estudo alcançou $51,7 \%$ de ganho relativo da ADM, próximo aos $57 \%$ observados por Boerboom et al. ${ }^{25}$

0 protocolo de reabilitação aplicado aos pacientes foi baseado na experiência do serviço e nos recursos disponíveis. Semelhante a Lindenhovius et al. ${ }^{9}$ os pacientes foram submetidos aos exercícios de alongamento ativo e passivo leve durante as sessões de terapia ocupacional, e foram orientados a realizá-los diariamente em casa. Diferentemente de Tan et al. ${ }^{26}$ em nenhum momento foi realizada manipulação forçada, que aumenta o risco de formação de hematomas, de $\mathrm{OH}$, e de piora da dor e da rigidez. ${ }^{11}$

A técnica cirúrgica foi a mesma para todos os pacientes, sendo realizada por dois cirurgiões. Semelhante a Koh et al. ${ }^{22}$ a via utilizada foi a via posterior, devido à versatilidade de acesso a todos os compartimentos do cotovelo. Nenhum paciente evoluiu com instabilidade ao término da cirurgia ou teve necessidade de uso do fixador externo. Semelhantemente a Higgs et al. ${ }^{14}$ a mobilização articular se iniciou no primeiro dia pós-operatório com o uso do MPC, mas sem o uso de cateter para bloqueio contínuo do plexo braquial. Os pacientes toleraram a dor apenas com o uso de analgésicos e anti-inflamatórios, conseguindo fazer o uso do MPC sem intercorrências.

Apesar de alguns cirurgiões evitarem a liberação cirúrgica por via posterior alegando risco maior de complicações da ferida como deiscência, em revisão sistemática sobre complicações, Cai et al. ${ }^{27}$ não observaram tal relação. Observamos apenas $1(6,7 \%)$ caso de complicação da ferida, sendo uma infecção superficial tratada com antibioticoterapia por via oral por 7 dias. Já Tan et al. ${ }^{26}$ que utiliazaram as vias medial e/ou lateral, e relataram 3 casos (5,8\%) de complicações da ferida, mas com infecção profunda e necessidade de limpeza cirúrgica, desbridamento e antibioticoterapia por via endovenosa.

Foram realizadas neurólise e transposição anterior do nervo ulnar em todos os casos do grupo cirúrgico, a fim de se evitar lesão do nervo ulnar ou eventual síndrome compressiva desse nervo no período pós-operatório. Nosso estudo apresentou quatro casos de neuropraxia transitória do nervo ulnar com melhora completa dos sintomas. Não há um consenso na literatura sobre qual é a melhor abordagem em relação ao nervo ulnar na RPTC.

Nosso estudo tem como principais vantagens a descrição de uma técnica e de uma reabilitação padronizada e de baixo custo, com o uso de escalas e questionários clínicos em tempos padronizados. Também avaliamos a ADM de flexoextensão do cotovelo de diversas maneiras, com a ADM, ganho da ADM, ganho relativo da ADM, extensão e flexão máximas, o que permite uma melhor comparação com outros estudos.
Entre as limitações deste estudo, o período de 6 meses pode ser considerado curto, mas estudos demonstram não haver diferenças estatisticamente significativas na ADM do cotovelo a partir desse período no tratamento da RPTC. ${ }^{17,28,29}$ O seguimento dos pacientes continuará até completar cinco anos do início do tratamento, para maior compreensão da relação do tempo pós-tratamento com a ADM do cotovelo e as escalas funcionais. Outra limitação é o fato de o tamanho da amostra ser relativamente pequeno, o que limitou a análise dos desfechos secundários.

\section{Conclusão}

A liberação cirúrgica do cotovelo associada ao protocolo de reabilitação é uma técnica segura, em que são obtidos resultados satisfatórios, com ganho absoluto de ADM de flexoextensão de $40^{\circ}$ e ganho relativo de $51,7 \%$, com uma baixa taxa de complicações.

\section{Conflito de Interesses}

Os autores declaram não haver conflito de interesses.

\section{Referências}

1 Morrey BF, An K-N. Functional Evaluation of the Elbow [Internet]. Morrey's The Elbow and Its Disorders, 2009:80-91. Available from: http://dx.doi.org/10.1016/b978-1-4160-2902-1.50010-3

2 Morrey BF, Askew LJ, Chao EY. A biomechanical study of normal functional elbow motion. J Bone Joint Surg Am 1981;63(06):872-877

3 Sardelli M, Tashjian RZ, MacWilliams BA. Functional elbow range of motion for contemporary tasks. J Bone Joint Surg Am 2011;93 (05):471-477

4 Cefo I, Eygendaal D. Arthroscopic arthrolysis for posttraumatic elbow stiffness. J Shoulder Elbow Surg 2011;20(03):434-439

5 Gundlach U, Eygendaal D. Surgical treatment of posttraumatic stiffness of the elbow: 2-year outcome in 21 patients after a column procedure. Acta Orthop 2008;79(01):74-77

6 Kodde IF, van Rijn J, van den Bekerom MPJ, Eygendaal D. Surgical treatment of post-traumatic elbow stiffness: a systematic review. J Shoulder Elbow Surg 2013;22(04):574-580

7 Chen HW, Liu GD, Wu LJ. Complications of treating terrible triad injury of the elbow: a systematic review. PLoS One 2014;9(05):e97476

8 Myden C, Hildebrand K. Elbow joint contracture after traumatic injury. J Shoulder Elbow Surg 2011;20(01):39-44

9 Lindenhovius AL, Doornberg JN, Ring D, Jupiter JB. Health status after open elbow contracture release. J Bone Joint Surg Am 2010; 92(12):2187-2195

10 Urbaniak JR, Hansen PE, Beissinger SF, Aitken MS. Correction of post-traumatic flexion contracture of the elbow by anterior capsulotomy. J Bone Joint Surg Am 1985;67(08):1160-1164

11 Itoh Y, Saegusa K, Ishiguro T, Horiuchi Y, Sasaki T, Uchinishi K. Operation for the stiff elbow. Int Orthop 1989;13(04):263-268

12 Søjbjerg JO, Kj-rsgaard-Andersen P, Johanssen HV, Sneppen O. Release of the stiff elbow followed by continuous passive motion and indomethacin treatment. J Shoulder Elbow Surg 1995;4:S20

13 Müller AM, Sadoghi P, Lucas R, et al. Effectiveness of bracing in the treatment of nonosseous restriction of elbow mobility: a systematic review and meta-analysis of 13 studies. J Shoulder Elbow Surg 2013;22(08):1146-1152

14 Higgs ZCJ, Danks BA, Sibinski M, Rymaszewski LA. Outcomes of open arthrolysis of the elbow without post-operative passive stretching. J Bone Joint Surg Br 2012;94(03):348-352

15 Morrey BF. Distraction arthroplasty. Clinical applications. Clin Orthop Relat Res 1993;(293):46-54 
578 Tratamento cirúrgico da rigidez pós-traumática do cotovelo Gracitelli et al.

16 Júnior JC, Zabeu JLA, Junior IA, Mattos CA, Myrrha JP. Arthroscopic treatment of post-traumatic elbow stiffness. Rev Bras Ortop 2015; 47(03):325-329

17 Park MJ, Chang MJ, Lee YB, Kang HJ. Surgical release for posttraumatic loss of elbow flexion. J Bone Joint Surg Am 2010;92(16):2692-2699

18 Morrey BF, Adams RA. Semiconstrained arthroplasty for the treatment of rheumatoid arthritis of the elbow. J Bone Joint Surg Am 1992;74(04):479-490

19 Orfale AG, Araújo PM, Ferraz MB, Natour J. Translation into Brazilian Portuguese, cultural adaptation and evaluation of the reliability of the Disabilities of the Arm, Shoulder and Hand Questionnaire. Braz J Med Biol Res 2005;38(02):293-302

20 Cauchoix J, Deburge A. [Elbow arthrolysis in post-traumatic rigidities]. Acta Orthop Belg 1975;41(04):385-392

21 Rettig LA, Hastings H II, Feinberg JR. Primary osteoarthritis of the elbow: lack of radiographic evidence for morphologic predisposition, results of operative debridement at intermediate follow-up, and basis for a new radiographic classification system. J Shoulder Elbow Surg 2008;17(01):97-105

22 Koh KH, Lim TK, Lee HI, Park MJ. Surgical release of elbow stiffness after internal fixation of intercondylar fracture of the distal humerus. J Shoulder Elbow Surg 2013;22(02):268-274
23 Miyazaki AN, Fregoneze M, Santos PD, et al. Avaliação dos resultados do tratamento cirúrgico da rigidez pós-traumática do cotovelo de pacientes esqueleticamente maturos. Rev Bras Ortop 2010;45(06):529-537

24 Ayadi D, Etienne P, Burny F, Schuind F. Results of open arthrolysis for elbow stiffness. A series of 22 cases. Acta Orthop Belg 2011;77 (04):453-457

25 Boerboom AL, de Meyier HE, Verburg AD, Verhaar JA. Arthrolysis for post-traumatic stiffness of the elbow. Int Orthop 1993;17(06): 346-349

26 Tan V, Daluiski A, Simic P, Hotchkiss RN. Outcome of open release for post-traumatic elbow stiffness. J Trauma 2006;61(03): 673-678

27 Cai J, Wang W, Yan H, et al. Complications of Open Elbow Arthrolysis in Post-Traumatic Elbow Stiffness: A Systematic Review. PLoS One 2015;10(09):e0138547

28 Aldridge JM III, Atkins TA, Gunneson EE, Urbaniak JR. Anterior release of the elbow for extension loss. J Bone Joint Surg Am 2004; 86(09):1955-1960

29 Stans AA, Maritz NG, O'Driscoll SW, Morrey BF. Operative treatment of elbow contracture in patients twenty-one years of age or younger. J Bone Joint Surg Am 2002;84(03):382-387 